This item was submitted to Loughborough's Research Repository by the author.

Items in Figshare are protected by copyright, with all rights reserved, unless otherwise indicated.

\title{
Visualisation of electronic excited-state correlation in real space
}

PLEASE CITE THE PUBLISHED VERSION

https://doi.org/10.1002/cptc.201900014

PUBLISHER

(c) Wiley

VERSION

AM (Accepted Manuscript)

PUBLISHER STATEMENT

This is the peer reviewed version of the following article: PLASSER, F., 2019. Visualisation of electronic excited-state correlation in real space. Chemphotochem, 3 (9), pp.702-706, which has been published in final form at https://doi.org/10.1002/cptc.201900014. This article may be used for non-commercial purposes in accordance with Wiley Terms and Conditions for Use of Self-Archived Versions.

\section{LICENCE}

CC BY-NC-ND 4.0

\section{REPOSITORY RECORD}

Plasser, Felix. 2019. "Visualisation of Electronic Excited-state Correlation in Real Space”. figshare. https://hdl.handle.net/2134/37141. 


\title{
Visualisation of Electronic Excited-State Correlation in Real Space
}

\author{
Dr Felix Plasser*
}

\begin{abstract}
A method for the visualisation of excited-state electron correlation is introduced and shown to address two notorious problems in excitedstate electronic structure theory, the analysis of excitonic correlation and the distinction between covalent and ionic wavefunction character. The method operates by representing the excited state in terms of electron and hole quasiparticles, fixing the hole on a fragment of the system and observing the resulting conditional electron density in real space. The application of this approach to oligothiophene, an exemplary conjugated polymer, illuminates excitonic correlation effects of its excited states in unprecedented clarity and detail. A study of naphthalene shows that the distinction between the ionic and covalent states of this molecule, which has so far only been achieved using elaborate valence-bond theory protocols, arises naturally in terms of electron-hole avoidance and enhanced overlap, respectively. More generally, the method is relevant for any excited state that cannot be described by a single electronic configuration.
\end{abstract}

Electronically excited states of molecules form the underpinnings of a wide range of processes of utmost scientific interest, such as light-driven natural processes [1, 2] photochemical reactions [3], signalling and sensing [4], and the operation of organoelectronic materials $[5,6]$. Computational photochemistry has become an indispensable part of scientific investigations in these areas through two main tasks, the interpretation of experimental results and the prediction of unknown photophysical properties. Indeed, the predictive power of computational photochemistry has steadily increased over the recent years due to the tremendous effort spent in the development of

*Department of Chemistry, Loughborough University, Loughborough, LE11 3TU, United Kingdom new methods as well as rapid progress in computer technology $[7,8,9,10,11,12]$. However, the interpretation of this wealth of computational data can act as a significant impediment in practical work. Therefore, a number of analysis tools have been developed to quantify excited-state properties $[13,14,15,16,17]$ and to visualise the molecular orbitals (MOs) involved and their location in space in a compact and rigorous way $[18,19,20,21,22,23]$.

However, the above-mentioned visualisation tools - and the MO picture itself — break down if the information of interest does not lie in the MOs themselves but in the interaction of different quasidegenerate electronic configurations. Such cases are widespread and two notorious failures of the MO picture relate to excitons in extended systems $[24,25,26]$ and to ionic/covalent wavefunction character in alternant hydrocarbons $[27,28]$. The first case is related to the emergence of band structure in large systems, which leads to excited states formed as a superposition of many different electronic configurations whose description requires moving from the MO picture to a representation in terms of correlated electron and hole quasiparticles [29, 26, 30, 31]. Previous attempts of visualising the ensuing correlated electron-hole distribution have relied on coarse-grained correlation plots $[32,33,34,35,36,37,38]$ and other abstract visualisation techniques $[39,40]$ but the goal of visualising correlated wavefunctions in real space has remained elusive. The second case derives from quasidegeneracies induced by approximate symmetries [41] and plays a role even for common smaller molecules such as butadiene, benzene, and naphthalene $[27,28]$. These molecules possess two distinct classes of states, denoted "+" and "-" [41], which are understood as ionic and covalent states within valence bond (VB) theory $[42,27]$. This distinction 
has existed since the early days of molecular quantum mechanics [41] but the quantification of covalent and ionic character is still quite cumbersome requiring specialised VB protocols [43] and has remained an active research topic [28]. This is particularly troublesome as it has been mentioned repeatedly that the ionic/covalent character has a strong but hard-to-pindown impact on commonly used electronic structure methods such as multiconfigurational self-consistent field $[44,45,46,47]$ and time-dependent density functional theory (TDDFT) $[48,49,50,51]$.

It is the purpose of this Communication to introduce a generally applicable and intuitive method for the analysis of excited-state correlation effects and to exemplify its power in the above-mentioned cases of exciton correlation and ionic/covalent wavefunction character. As a first step, high-level excited-state computations are performed using either TDDFT or wavefunction-based ab initio approaches. Subsequently, the involved states are represented in the more compact form of two-body electron-hole distributions. The central quantity, here, is the oneelectron transition density matrix (1TDM) [34, 52, $22,53]$, which in coordinate space is given as

$$
\gamma_{0 I}\left(r_{h}, r_{e}\right)=\sum_{p q}\left\langle\Psi_{0}\left|\hat{a}_{p}^{\dagger} \hat{a}_{q}\right| \Psi_{I}\right\rangle \phi_{p}^{*}\left(r_{h}\right) \phi_{q}\left(r_{e}\right) .
$$

$\Psi_{0}$ and $\Psi_{I}$ are the many-body wavefunctions of the ground and excited states, $\phi_{p}$ and $\phi_{q}$ are molecular orbitals, and $\hat{a}_{p}^{\dagger}$ and $\hat{a}_{q}$ are the associated creation and annihilation operators. The coordinates $r_{h}$ and $r_{e}$ refer to the spatial positions of the excitation hole and the excited electron, respectively, and $\gamma_{0 I}\left(r_{h}, r_{e}\right)$ can be viewed as the wavefunction of the electron-hole pair. The essential property of $\gamma_{0 I}\left(r_{h}, r_{e}\right)$ is that, as a two-body function, it represents a middle ground between the original many-body wavefunctions and the uncorrelated description in terms of MOs (i.e. one-body functions).

As a first option, it is possible to use the 1TDM to construct the overall densities of the excited electron $\rho_{e}$ and the excitation hole $\rho_{h}$ by integrating out either the hole or the electron coordinate [22], i.e.

$$
\rho_{e}\left(r_{e}\right)=\int\left|\gamma_{0 I}\left(r_{h}, r_{e}\right)\right|^{2} \mathrm{~d} r_{h}
$$

$$
\rho_{h}\left(r_{h}\right)=\int\left|\gamma_{0 I}\left(r_{h}, r_{e}\right)\right|^{2} \mathrm{~d} r_{e}
$$

These densities can be intuitively represented in real space showing the overall distribution of the excitation over the molecule but do not provide any information about correlation effects. An entirely different representation is given by the charge-transfer numbers $[52,37]$, which are formally obtained by a partial integral of the form [22]

$$
\Omega_{A B}=\int_{A} \int_{B}\left|\gamma_{0 I}\left(r_{h}, r_{e}\right)\right|^{2} \mathrm{~d} r_{e} \mathrm{~d} r_{h}
$$

where $A$ and $B$ are two fragments of the system. Here, $\Omega_{A B}$ represents the probability that the hole is on fragment $A$ while the electron is on fragment $B$. The charge-transfer numbers go beyond the static description provided by $\rho_{e}$ and $\rho_{h}$ through encoding correlation effects between the electron and hole. However, they do so only in a very abstract way (see below).

Using the applied notation, the method proposed within this Communication can be written as a combination of Eqs (2) and (4) and we define the conditional electron density as

$$
\rho_{e}^{h: A}\left(r_{e}\right)=\int_{A}\left|\gamma^{0 I}\left(r_{h}, r_{e}\right)\right|^{2} \mathrm{~d} r_{h} .
$$

$\rho_{e}^{h: A}$ represents the probability distribution of the excited electron under the condition that the probe hole is located on fragment $A$. We will show below that this is an immensely powerful tool that provides intuitive insight into intricate excited-state correlation effects. To the best of our knowledge such a strategy has never been used for excited state analysis. However, it should be noted for completeness that the domain-averaged Fermi hole [54], used for analysing exchange effects and chemical bonding in the ground state, and a recently proposed analysis of spin correlation densities [55] follow similar ideas. Practically, $\Omega_{A B}$ and $\rho_{e}^{h: A}$ are evaluated in the spirit of a Löwdin population analysis $[56,57,58]$, see Section $\mathrm{S} 1$ of the Supporting Information (SI) for details. ${ }^{1}$

\footnotetext{
${ }^{1}$ See Supporting Information for matrix form of the employed equations (S1); frontier orbitals/transitions and further
} 
To illustrate the challenges present when analysing excited-states with previously available tools, we want to start with the example of oligothiophene, a widely studied system due to its importance as a building block for organoelectronic materials [59, 5 , $60,61,62,63]$. Computations were performed on the oligomer containing six thiophene units (OT6) using TDDFT in the Tamm-Dancoff approximation $[64,65,66]$ with the CAM-B3LYP functional $[67,68,69]$ and Ahlrichs' SV(P) basis set [70]. The symmetry labels and excitation energies of the lowest five states are presented in the left column of Figure 1. The lowest state is of $B_{u}$ symmetry, followed by two states of $A_{g}$ symmetry and to more $B_{u}$ states. In an attempt to learn more about these states, we first compute the electron and hole densities, as defined in Eqs (2) and (3) and plot them as isosurfaces $[71,72]$. An inspection of these densities, shown in the center column of Figure 1, reveals that all states possess about the same degree of delocalization and that similar nodal planes are present on the individual thiophene units. However, it is difficult to discern any differences between these plots and even more to find a physical explanation of these differences. Apparently, the description in terms of independent electron and hole densities is insufficient and we have to proceed to an analysis of correlation effects.

In order to get a glimpse into the involved correlation effects, we compute the charge-transfer numbers as defined in Eq. (4), where every thiophene monomer in OT6 represents one fragment $(A$ or $B)$. The charge-transfer numbers cannot be directly visualised in real space but one can represent them in terms of a pseudocolor matrix plot $[34,37,30]$ as shown on the right-hand side of Figure 1. Here, every box corresponds to one pair of fragments $A$ and $B$ and its shade represents the $\Omega_{A B}$ value. The main diagonal - going from lower left to upper right represents the cases where the electron and hole are on the same fragment while the off-diagonal elements correspond to charge transfer contributions. In this representation the different structure of the different

discussion for OT6 (S2); domain-based natural transition orbitals for OT6 (S3); frontier orbitals/transitions for naphthalene (S4). Molecular geometries, input and output files (QChem and TheoDORE) of the computations (zip).

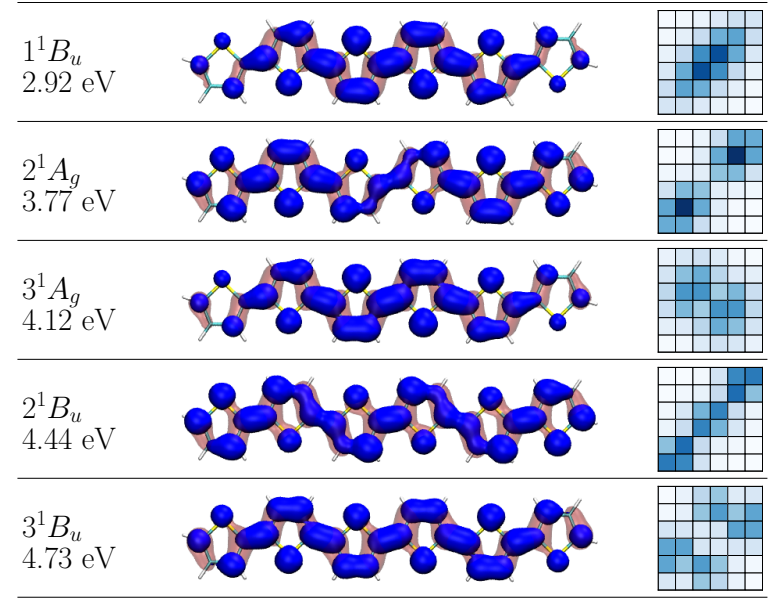

Figure 1: Analysis of the first five excited states of oligothiophene. Left: Symmetry label and excitation energy. Center: Densities of the excited electron (dark blue) and excitation hole (light red); the surfaces plotted encompass $75 \%$ of the total densities. Right: electron-hole correlation plots; the electron position is plotted from bottom to top, the hole from left to right.

states becomes much more apparent. $1^{1} B_{u}, 2^{1} A_{g}$, and $2^{1} B_{u}$ belong to one exciton band where the main contributions are on the diagonal, which means that a strongly bound exciton is present. $3^{1} A_{g}$ and $3^{1} B_{u}$ form a different band with enhanced dynamic charge transfer character. If the exciton quasi-particle is represented as a hydrogen atom in a box [26], then the first type of band corresponds to an $s$-orbital and the second band to a $p$-orbital of the hydrogen atom [30]. These will be denoted $S$ - and $P$-excitons in the following. In summary, the charge-transfer numbers provided a completely new way to classify the excited states of OT6, which was not apparent using either the electron and hole densities or the MOs (Table S2) but it did so only in a very abstract way.

As a final step, we compute the newly introduced conditional electron densities defined by Eq. (5). The probe hole was located on the three symmetry-unique thiophene units and the appropriate conditional elec- 


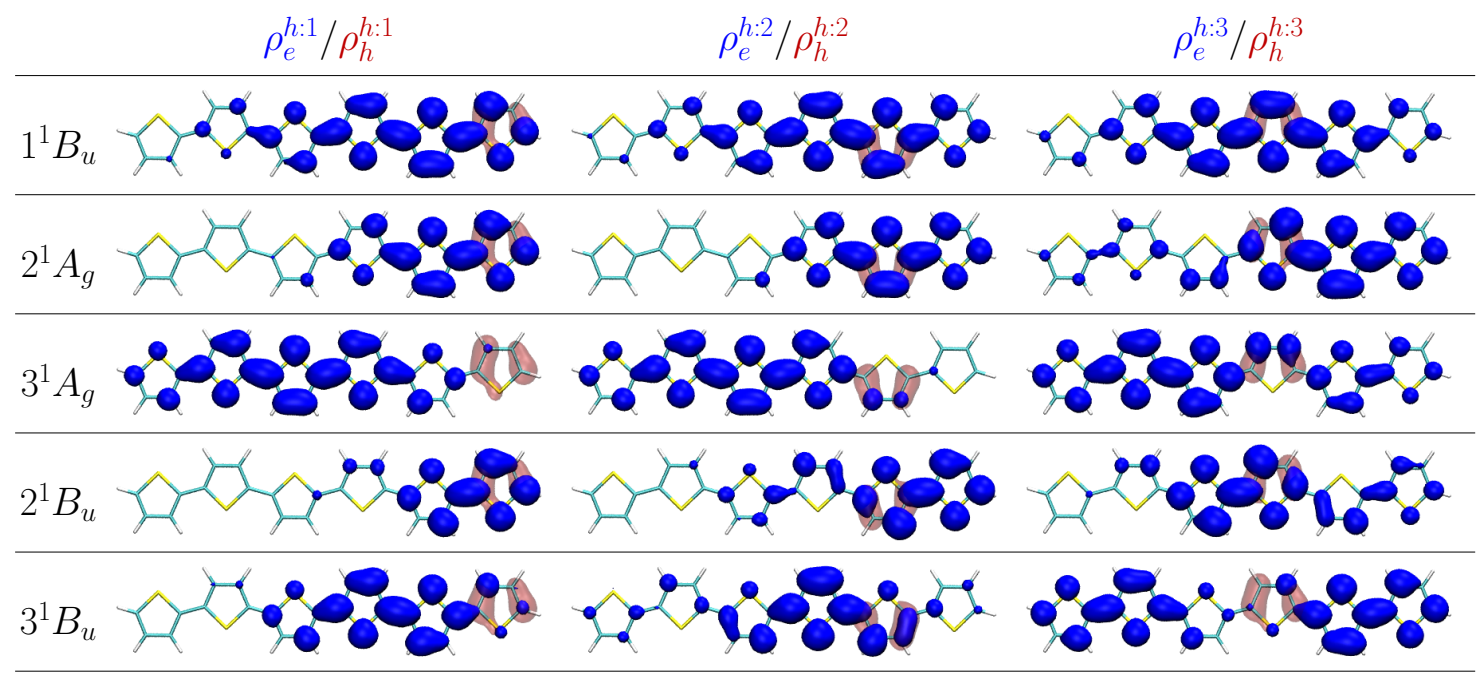

Figure 2: Conditional electron densities (dark blue) determined for the probe hole (light red) localised on different thiophene units computed for oligothiophene at the TDDFT/CAM-B3LYP level of theory. The surfaces plotted encompass $75 \%$ of the total electron densities.

tron density was plotted in real space, see Figure 2. Each of these plots corresponds to one column of the correlation plots in Figure 1. At first sight, the rich structure of these excited states becomes apparent. The uniform distributions in Figure 1 turn into a mix of different shapes. In the first column the densities are presented for the probe hole located on the rightmost thiophene. It is observed that in the case of the $S$-excitons $\left(1^{1} B_{u}, 2^{1} A_{g}, 2^{1} B_{u}\right)$ the electron is pulled to the right toward the hole and that this attraction becomes stronger for the higher states. By contrast, the electron is effectively pushed away from the hole for the $P$-excitons $\left(3^{1} A_{g}, 3^{1} B_{u}\right)$. Moving to the second and third columns in Figure 2 corresponds to moving the hole to the second and third thiophene unit from the right and observing how the conditional electron density adjusts to the new position. Whereas these adjustments are comparatively small for the first two states $\left(1^{1} B_{u}\right.$ and $\left.2^{1} A_{g}\right)$, a more intricate pattern is observed for the $3^{1} A_{g}$ state as the conditional electron density always avoids and surrounds the hole as the hole is moved through the system, and a nodal plane going through the sulphur atom is observed. The two following states, $2^{1} B_{u}$ and $3^{1} B_{u}$, have a somewhat more complicated structure as reflected by the enhanced number of nodal planes in the correlation plots of Figure 1. As detailed in the discussion below Table S2, the presented analysis provides a consistent description with our previously defined more abstract statistical descriptors, such as the exciton size [53], the electron-hole correlation coefficient [16, 31], and electron-hole entanglement [73] but offers insight into the underlying causes of the trends observed. Rather than plotting the conditional electron densities as a whole, it is also possible to compute the individual eigenfunctions of the respective density matrices in analogy to Ref. [74]. The resulting "domain-based natural transition orbitals" are shown in Figure S3 providing a similar albeit less compact description

As a second example, we consider the naphthalene molecule and compute its lowest eight singlet excited states using the algebraic diagrammic construction method $\operatorname{ADC}(2)[75,76,77,65]$. The conditional electron densities, determined for the probe hole located on two different atoms, are presented 
in Figure 3. Again, a rich structure and symmetry breaking effects are observed, notwithstanding the fact that the canonical orbitals involved (see Table S4) all obey the $D_{2 h}$ symmetry of the overall system. More specifically, two different cases are observed as the conditional electron density could either move toward or avoid the hole. The first case corresponds to enhanced overlap rendering the red hole barely visible in Figure 3 while the second means that the hole is unobstructed. The crucial observation is that the "_" states consistently correspond to electronhole avoidance whereas the "+" states correspond to strong electron-hole overlap. This observation opens an entirely new and unexpected viewpoint on the problem of ionic vs. covalent states: Rather than going through the involved framework of VB theory, they can be directly distinguished using the two-body electron-hole distribution.

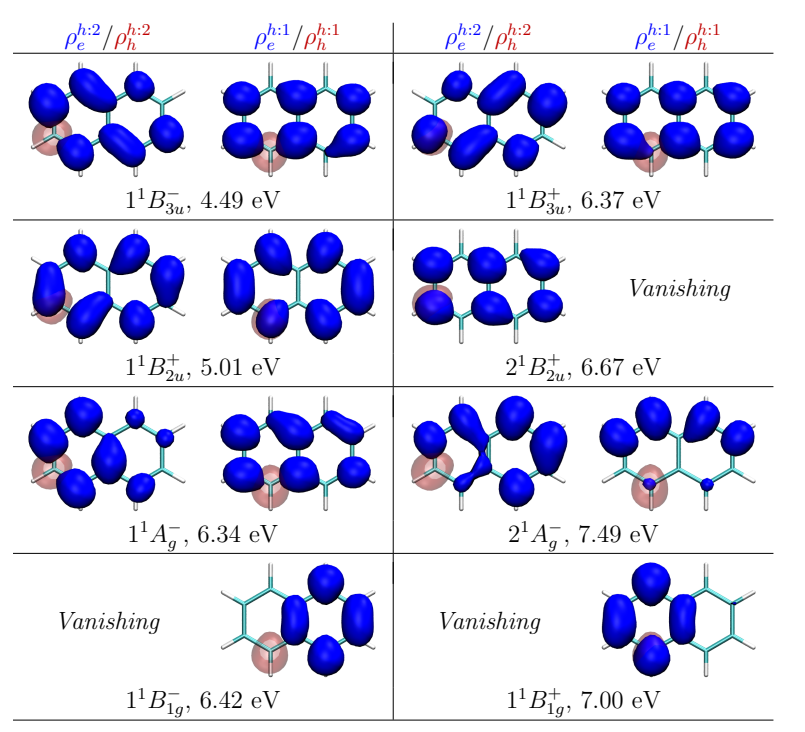

Figure 3: Conditional electron densities $\left(\rho_{e}^{h: A}\right.$, dark blue) for the eight lowest singlet excited states of napthalene determined for the probe hole $\left(\rho_{h}^{h: A}\right.$, light red) localised on two different atoms. The surfaces plotted encompass $75 \%$ of the total electron densities. Cases with vanishing Löwdin-populations of the hole on the probe atom $(<0.05 e)$ are not shown.
Aside from the general distinction between ionic and covalent states, also more detailed statements can be made about the excited states presented in Figure 3. The simplest case are the $B_{2 u}$ states (second row), which are both dominated by only a single electronic transition (HOMO $\rightarrow$ LUMO and HOMO$1 \rightarrow \mathrm{LUMO}+1$, respectively). As a consequence, correlation effects do not play a significant role and the conditional electron densities retain the overall symmetric shape of the total electron densities. For the $B_{3 u}$ states (first row) stronger correlations are observed but the effect is restricted to individual $\mathrm{C}$ atoms. The main depletion of conditional electron density always occurs for $\mathrm{C}$ atoms on opposing sides of the molecule. In the case of the $B_{1 g}$ states, on the other hand, the conditional electron density is completely restricted to one side of the molecule leaving the other side empty. This behaviour can be rationalised by considering that linear combinations of the involved orbitals (HOMO/HOMO-2 and LUMO/LUMO+2) will yield orbitals that are localised on either side of the molecule, cf. Ref [78]. Finally, the ${ }^{1} A_{g}^{-}$states show a somewhat more complicated pattern.

To understand the relation between the presented analysis and VB theory, it is first worth realising that a covalent structure in VB theory corresponds to two singlet-coupled open-shell electrons on neighbouring atoms $[45,78]$. In this work, the open-shell electrons are represented as the electron and hole quasiparticles, and these correctly occupy different locations in the covalent "_" states. Conversely, ionic states mean the presence of closed-shell electrons in localised orbitals on neighbouring atoms, which can also be represented as open-shell electrons present in overlapping delocalised orbitals [78]. This leads to the enhanced electron-hole overlap seen in Figure 3. To rationalise the energetics of the states involved, it is worth realising that a singlet-coupled electronhole pair experiences exchange repulsion as well as Coulomb attraction [24, 29, 31]. For the small naphthalene molecule exchange dominates and the lowest state is of repulsive nature. By contrast, in the larger OT6 system (Figure 2) the Coulomb attraction becomes more important and the lowest state is a bound exciton. 
In summary, a method for visualising excited-state correlation was presented and shown to provide detailed insight into two quite distinct effects, excitonic correlation and ionic/covalent wavefunction character. More generally, the method is relevant for any excited state that cannot be represented by a single electronic configuration or, more precisely, when the entanglement entropy between electron and hole [73] is non-vanishing. The described functionalities were implemented in the TheoDORE [79] program package, which operates by post-processing excited-state calculations and provides interfaces to a wide range of electronic structure methods and program packages. The evaluation of conditional electron densities is computationally cheap, easily applied and works for any electronic structure method supplying transition density matrices. Therefore, we believe that it will be a powerful addition to the computational photochemistry toolbox serving for a variety of tasks, such as revealing the properties of newly discovered or designed conjugated polymers [80, 81], studying the influence of structural and environmental effects on their excited states [82], and elucidating exciplex formation between biochromophores [83]. Whereas the present analysis focusses on a time-independent description of electron correlation, it would also be interesting to extend the concept to the description of attosecond dynamics $[84,85]$. The method can also be extended to the analysis of more general strong correlation phenomena [86] by substituting the two-body density matrix for the electron-hole distribution.

\section{Acknowledgments}

The author thanks G. Hermann for writing the interface between TheoDORE and orbkit used here and gratefully acknowledges Loughborough University for startup funding. The author thanks I. A. Wright, S. A. Mewes, J.-M. Mewes, and A. Dreuw for comments on the manuscript.

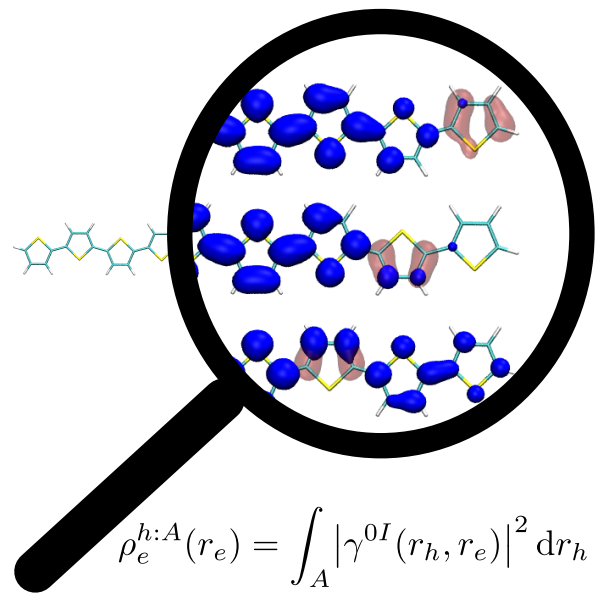

Figure 4: TOC figure.

\section{Keywords}

Quantum chemistry, excited states, wavefunction analysis

\section{TOC}

New insight: A method for analysing excited-state correlation is introduced and used for illuminating excitonic correlation effects in a conjugated oligomer and for differentiating between ionic and covalent wavefunction character in naphthalene.

\section{References}

[1] F. Plasser, A. J. A. Aquino, H. Lischka, and D. Nachtigallova. Top. Curr. Chem. 2015. 356, $1-38$.

[2] A. Chenu and G. D. Scholes. Annu. Rev. Phys. Chem. 2015. 66, 69-96.

[3] N. Hoffmann. Chem. Rev. 2008. 108, 10521103.

[4] S. J. Butler and D. Parker. Chem. Soc. Rev. 2013. 42, 1652-1666. 
[5] G. Gigli, G. Barbarella, L. Favaretto, F. Cacialli, and R. Cingolani. Appl. Phys. Lett. 1999. 75, 439-441.

[6] M. Kuik, G.-J. A. H. Wetzelaer, H. T. Nicolai, N. I. Craciun, D. M. De Leeuw, and P. W. M. Blom. Adv. Mater. 2014. 26, 512-31.

[7] A. Dreuw and M. Head-Gordon. Chem. Rev. 2005. 105, 4009-4037.

[8] A. I. Krylov. Annu. Rev. Phys. Chem. 2008. 59, 433-62.

[9] F. Plasser, M. Barbatti, A. J. A. Aquino, and H. Lischka. Theor. Chem. Acc. 2012. 131, 1073.

[10] L. González, D. Escudero, and L. SerranoAndrés. ChemPhysChem 2012. 13, 28-51.

[11] M. Casida and M. Huix-Rotllant. Annu. Rev. Phys. Chem. 2012. 63, 287-323.

[12] J. M. Herbert, X. Zhang, A. F. Morrison, and J. Liu. Acc. Chem. Res. 2016. 49, 931-941.

[13] T. Etienne, X. Assfeld, and A. Monari. J. Chem. Theory Comput. 2014. 10, 3906-3914.

[14] S. Matsika, X. Feng, A. V. Luzanov, and A. I. Krylov. J. Phys. Chem. A 2014. 118, 11943-55.

[15] C. Adamo, T. Le Bahers, M. Savarese, L. Wilbraham, G. Garcia, R. Fukuda, M. Ehara, N. Rega, and I. Ciofini. Coord. Chem. Rev. 2015. 304-305, 166-178.

[16] F. Plasser, B. Thomitzni, S. A. Bäppler, J. Wenzel, D. R. Rehn, M. Wormit, and A. Dreuw. J. Comp. Chem. 2015. 36, 1609-1620.

[17] G. M. J. Barca, A. T. B. Gilbert, and P. M. W. Gill. J. Chem. Theory Comput. 2018. 14, 9-13.

[18] A. V. Luzanov, A. A. Sukhorukov, and V. E. Umanskii. Theoretical and Experimental Chemistry 1976. 10, 354-361.

[19] M. Head-Gordon, A. M. Grana, D. Maurice, and C. A. White. J. Chem. Phys. 1995. 99, 1426114270 .
[20] R. L. Martin. J. Chem. Phys. 2003. 118, 47754777 .

[21] I. Mayer. Chem. Phys. Lett. 2007. 437, 284-286.

[22] F. Plasser, M. Wormit, and A. Dreuw. J. Chem. Phys. 2014. 141, 024106.

[23] T. Etienne. J. Chem. Phys. 2015. 142, 244103.

[24] G. D. Scholes and G. Rumbles. Nature Materials 2006. 5, 683-96.

[25] B. M. Savoie, N. E. Jackson, T. J. Marks, and M. A. Ratner. Phys. Chem. Chem. Phys. 2013. 15, 4538-47.

[26] W. Barford. J. Phys. Chem. A 2013. 117, 266571.

[27] T. Hashimoto, H. Nakano, and K. Hirao. J. Chem. Phys. 1996. 104, 6244-6258.

[28] J. Gu, W. Wu, D. Danovich, R. Hoffmann, Y. Tsuji, and S. Shaik. J. Am. Chem. Soc. 2017. 139, 9302-9316.

[29] S. Brazovskii and N. Kirova. Chem. Soc. Rev. 2010. 39, 2453.

[30] S. Mewes, J. Mewes, A. Dreuw, and F. Plasser. PCCP 2016. 18, 2548-2563.

[31] S. A. Mewes, F. Plasser, and A. Dreuw. J. Phys. Chem. Lett. 2017. 8, 1205-1210.

[32] E. Zojer, P. Buchacher, F. Wudl, J. Cornil, J. P. Calbert, J. L. Brédas, and G. Leising. J. Chem. Phys. 2000. 113, 10002-10012.

[33] J. Rissler, H. Bässler, F. Gebhard, and P. Schwerdtfeger. Phys. Rev. B 2001. 64, 045122.

[34] S. Tretiak and S. Mukamel. Chem. Rev. 2002. 102, 3171-3212.

[35] J. Cornil, I. Gueli, A. Dkhissi, J. C. SanchoGarcia, E. Hennebicq, J. P. Calbert, V. Lemaur, D. Beljonne, and J. L. Brédas. J. Chem. Phys. 2003. 118, 6615-6623. 
[36] B. M. Wong. J. Phys. Chem. C. Nanomater. Interfaces 2009. 113, 21921-21927.

[37] F. Plasser and H. Lischka. J. Chem. Theory Comput. 2012. 8, 2777-2789.

[38] Y. Li and C. A. Ullrich. J. Chem. Theory Comput. 2015. 11, 5838-5852.

[39] M. Rohlfing and S. G. Louie. Phys. Rev. Lett. 1999. 82, 1959-1962.

[40] W. Barford and N. Paiboonvorachat. J. Chem. Phys. 2008. 129, 164716.

[41] R. Pariser. J. Chem. Phys. 1956. 24, 250-268.

[42] K. Schulten, I. Ohmine, and M. Karplus. J. Chem. Phys. 1976. 64, 4422-4441.

[43] K. Hirao, H. Nakano, and K. Nakayama. J. Chem. Phys. 1997. 107, 9966-9974.

[44] B. O. Roos, K. Andersson, and M. P. Fülscher. Chem. Phys. Lett. 1992. 192, 5-13.

[45] W. T. Borden and E. R. Davidson. Acc. Chem. Res. 1996. 29, 67-75.

[46] C. Angeli. J. Comput. Chem. 2009. 30, 13191333.

[47] H. Lischka, D. Nachtigallová, A. J. A. Aquino, P. Szalay, F. Plasser, F. B. C. Machado, and M. Barbatti. Chem. Rev. 2018. 118, 7293-7361.

[48] S. Grimme and M. Parac. ChemPhysChem 2003. 4, 292-5.

[49] R. M. Richard and J. M. Herbert. J. Chem. Theory Comput. 2011. 7, 1296-1306.

[50] N. Kuritz, T. Stein, R. Baer, and L. Kronik. J. Chem. Theory Comput 2011. 7, 2408-2415.

[51] A. Prlj, M. E. Sandoval-Salinas, D. Casanova, D. Jacquemin, and C. Corminboeuf. J. Chem. Theory Comput. 2016. 12, 2652-2660.

[52] A. V. Luzanov and O. A. Zhikol. Int. J. Quantum Chem. 2010. 110, 902-924.
[53] S. A. Bäppler, F. Plasser, M. Wormit, and A. Dreuw. Phys. Rev. A 2014. 90, 052521.

[54] R. Ponec and D. L. Cooper. Faraday Discuss. 2007. 135, 31-42.

[55] J. B. Schriber, K. P. Hannon, C. Li, and F. A. Evangelista. J. Chem. Theory Comput. 2018. 14, 6295-6305.

[56] P. O. Löwdin. J. Chem. Phys. 1950. 18, 365375.

[57] A. A. Voityuk. J. Chem. Phys. 2014. 140, 244117.

[58] S. Mai, F. Plasser, J. Dorn, M. Fumanal, C. Daniel, and L. González. Coord. Chem. Rev. 2018. 361, $74-97$.

[59] P. Bäuerle, T. Fischer, B. Bidlingmeier, A. Stabel, and J. P. Rabe. Angew. Chemie 1995. 10\%, 335-339.

[60] H. Tamura, R. Martinazzo, M. Ruckenbauer, and I. Burghardt. J. Chem. Phys. 2012. 13\%, $22 \mathrm{~A} 540$.

[61] H. Li, R. Nieman, A. J. A. Aquino, H. Lischka, and S. Tretiak. J. Chem. Theory Comput. 2014. 10, 3280-3289.

[62] S. Kraner, R. Scholz, F. Plasser, C. Koerner, and K. Leo. J. Chem. Phys. 2015. 143, 244905.

[63] B. Holzer, J. Bintinger, D. Lumpi, C. Choi, Y. Kim, B. Stöger, C. Hametner, M. MarchettiDeschmann, F. Plasser, E. Horkel, I. Kymissis, and J. Fröhlich. ChemPhys Chem 2017. 18, 549 563.

[64] S. Hirata and M. Head-Gordon. Chem. Phys. Lett. 1999. 314, 291-299.

[65] Y. Shao and et al. Mol. Phys. 2015. 113, 184215.

[66] S. A. Mewes, F. Plasser, and A. Dreuw. J. Chem. Phys. 2015. 143, 171101. 
[67] A. D. Becke. J. Chem. Phys. 1993. 98, 56485652 .

[68] C. Lee, W. Yang, and R. G. Parr. Phys. Rev. B 1988. 37, 785-789.

[69] T. Yanai, D. P. Tew, and N. C. Handy. Chem. Phys. Lett. 2004. 393, 51-57.

[70] A. Schafer, H. Horn, and R. Ahlrichs. J. Chem. Phys. 1992. 97, 2571-2577.

[71] G. Hermann, V. Pohl, J. C. Tremblay, B. Paulus, H. C. Hege, and A. Schild. J. Comput. Chem. 2016. 37, 1511-1520.

[72] W. Humphrey, A. Dalke, and K. Schulten. J. of Mol. Graphics 1996. 14, $33-38$.

[73] F. Plasser. J. Chem. Phys. 2016. 144, 194107.

[74] P. Bultinck, D. L. Cooper, and R. Ponec. J. Phys. Chem. A 2010. 114, 8754-8763.

[75] J. Schirmer. Phys. Rev. A 1982. 26, 2395-2416.

[76] M. Wormit, D. R. Rehn, P. H. Harbach, J. Wenzel, C. M. Krauter, E. Epifanovsky, and A. Dreuw. Mol. Phys. 2014. 112, 774-784.

[77] A. Dreuw and M. Wormit. WIREs: Comp. Mol. Sci. 2015. 5, 82-95.

[78] Y. Yang, E. R. Davidson, and W. Yang. PNAS 2016. 113, E5098-E5107.

[79] F. Plasser. TheoDORE: a package for theoretical density, orbital relaxation, and exciton analysis; available from http://theodore-qc. sourceforge.net/.

[80] T. Rosenau, A. Potthast, N. S. Zwirchmayr, H. Hettegger, F. Plasser, T. Hosoya, M. Bacher, K. Krainz, and T. Dietz. Cellulose 2017. 24, 3671-3687.

[81] S. Kraner, R. Scholz, C. Koerner, and K. Leo. J. Phys. Chem. C 2015. 119, 22820-22825.

[82] H. Ma and A. Troisi. Adv. Chem. Phys. 2014. 26, 6163-6167.
[83] F. Plasser and H. Lischka. Photochem. Photobiol. Sci. 2013. 12, 1440-52.

[84] J. Feist, S. Nagele, R. Pazourek, E. Persson, B. I. Schneider, L. A. Collins, and J. Burgdörfer. Phys. Rev. Lett. 2009. 103, 063002.

[85] A. D. Dutoi. Mol. Phys. 2014. 112, 1-11.

[86] F. Plasser, H. Pašalic, M. H. Gerzabek, F. Libisch, R. Reiter, J. Burgdörfer, T. Müller, R. Shepard, and H. Lischka. Angew. Chem., Int. Ed. 2013. 52, 2581-2584. 